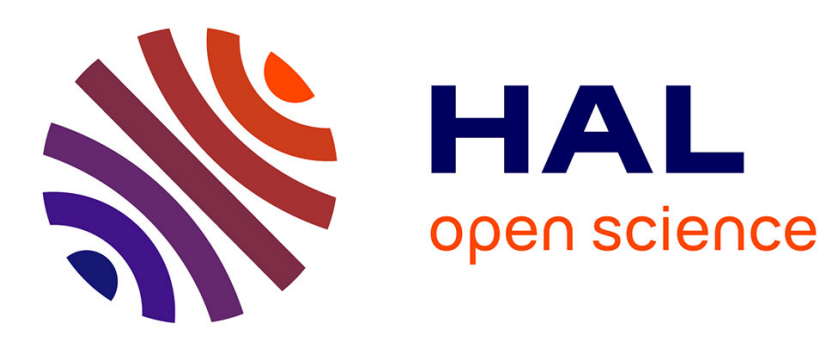

\title{
$80 \mathrm{GHz}$ waveform generated by the optical Fourier synthesis of four spectral sidebands
}

Julien Fatome, Kamal Hammani, Bertrand Kibler, Christophe Finot

\section{To cite this version:}

Julien Fatome, Kamal Hammani, Bertrand Kibler, Christophe Finot. $80 \mathrm{GHz}$ waveform generated by the optical Fourier synthesis of four spectral sidebands. Laser Physics Letters, 2016, 13 (1), pp.015102. 10.1088/1612-2011/13/1/015102 . hal-01238866

\section{HAL Id: hal-01238866 https://hal.science/hal-01238866}

Submitted on 8 Dec 2015

HAL is a multi-disciplinary open access archive for the deposit and dissemination of scientific research documents, whether they are published or not. The documents may come from teaching and research institutions in France or abroad, or from public or private research centers.
L'archive ouverte pluridisciplinaire HAL, est destinée au dépôt et à la diffusion de documents scientifiques de niveau recherche, publiés ou non, émanant des établissements d'enseignement et de recherche français ou étrangers, des laboratoires publics ou privés. 


\title{
80-GHz waveform generator by optical Fourier synthesis of four spectral sidebands
}

\author{
Julien Fatome, Kamal Hammani, Bertrand Kibler \& Christophe Finot \\ Laboratoire Interdisciplinaire Carnot de Bourgogne, UMR 6303 CNRS-Université de \\ Bourgogne Franche-Comté, 9 avenue Alain Savary, BP 47870, 21078 Dijon Cedex, France \\ E-mail: christophe.finot@u-bourgogne.fr
}

August 2015

\begin{abstract}
Using linear phase shaping of a simple 4-line optical frequency comb, we experimentally demonstrate the generation of various optical waveforms such as parabolic, triangular or flat-top pulse trains at a repetition rate of $80 \mathrm{GHz}$. The initial $80-\mathrm{GHz}$ comb is obtained through nonlinear spectral broadening of a $40-\mathrm{GHz}$ carrier-suppressed sinusoidal beating in a highly nonlinear fiber. Proof-of-principle experiments are reported for two distinct configurations of the waveform generator: continuous trains and bunches of shaped pulses.
\end{abstract}

PACS numbers: 42.65.Re, 42.65.Wi

Keywords: Optical pulse shaping, nonlinear fiber optics, waveform generation

Submitted to: Laser Phys. Lett. 


\section{Introduction}

Versatile and easy to implement methods to generate arbitrary optical waveforms at high repetition rates are of considerable interest with applications in optical communications, all-optical signal processing, instrumentation systems and microwave signal manipulation [1,2]. While shaping sinusoidal, Gaussian or hyperbolic secant intensity profiles is commonly achieved by means of modulators or mode-locked lasers, other pulse profiles such as parabolic, triangular or flat-top shapes still remain challenging to synthesize. In this context, several strategies were already explored. First, the linear pulse shaping is a common method to carve an initial ultrashort pulse train into the desired shape. In this case, the transfer function is thus given in the spectral domain by the ratio between the target field and the initial optical field. The linear shaping of picosecond pulses was demonstrated by means of numerous components including spatial light modulators [3,4], superstructured fiber Bragg gratings [5], acousto-optics devices [6] or arrayed waveguide gratings [7]. Furthermore, the line-byline shaping of a coherent frequency comb made of tens of spectral components was also investigated to generate more complex structures [8] whereas Fourier synthesis of a few discrete frequencies spectrum was exploited to efficiently generate high-fidelity ultrafast periodic intensity profiles [9-11].

Besides linear shaping techniques, several nonlinear methods were implemented to benefit from the adiabatic evolution of the intensity pulse profile upon propagation in optical fibers. For instance, high-quality parabolic intensity profiles [12] or triangular shapes [13, 14] were generated thanks to the combined effects of Kerr nonlinearity and normal dispersion. Other examples of efficient methods are based on the photonic generation involving specific Mach-Zehnder modulators [15, 16], microwave photonic filters [17] as well as frequencyto-time conversion $[18,19]$.

Recently, we theoretically and experimentally demonstrated a new linear approach enabling the synthesis of periodic high-repetition rate pulses with various intensity profiles ranging from parabola to triangular and flat-top pulses [20]. By using only four comb lines resulting from a phase-modulated continuous wave, we successfully demonstrated the generation of high-fidelity ultrafast periodic waveforms at $40 \mathrm{GHz}$. In the present work, after recalling the principle of operation of this approach, we describe a new scenario to obtain the required initial spectrum by taking advantage of the four-wave mixing process occurring in a highly nonlinear fiber (HNLF). This concept is experimentally validated at a repetition rate of $80-\mathrm{GHz}$ and the generation of bunch of shaped pulses is then investigated.

\section{Principle of operation}

\subsection{Waveform synthesis based on four spectral lines}

When considering a temporal train of symmetric pulses, the corresponding 4-band optical spectrum is expected to be also symmetric and only two parameters are therefore required to mathematically describe such a spectrum. As illustrated in figure 1, the first parameter is the ratio $A$ between the amplitude of the central components and the amplitude of lateral sidebands. The second parameter consists in the spectral phase $\varphi$ of the lateral sidebands relative to the central components.

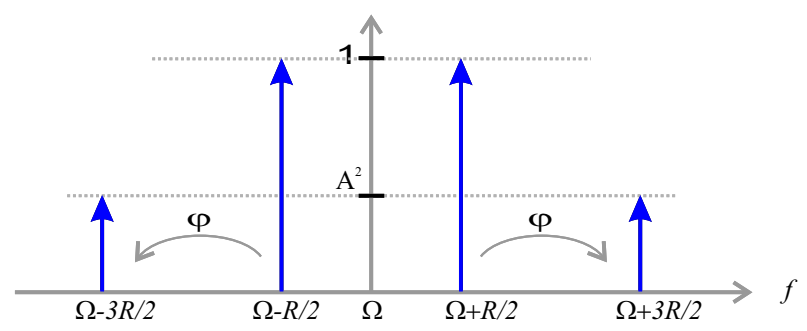

Figure 1. Optical spectrum of a four band model. $\Omega$ is the carrier frequency and $R$ is the repetition rate.

From this particular spectrum, it is easy to retrieve the analytical expression of the temporal intensity profile [20] given by :

$$
I(t)=2\left[1+A^{2}+(1+2 A \cos (\varphi)) \cos \left(\omega_{0} t\right)+\right.
$$$$
\left.2 A \cos (\varphi) \cos \left(2 \omega_{0} t\right)+A^{2} \cos \left(3 \omega_{0} t\right)\right]
$$

with $\omega_{0}=2 \pi R, R$ being the repetition rate of the pulse train. By exploring the bidimensional space $\left(A^{2}, \varphi\right)$ (figure 2(a)), we notice that the degree of kurtosis excess [21] calculated over one period of the generated pulse train varies significantly. It may reach positive or negative values. This implies that very different levels of peakedness can be reached, allowing to synthesize numerous temporal intensity shape profiles. Indeed we previously demonstrated that operating at a value $A^{2}$ of $-12 \mathrm{~dB}$ was particularly appealing as it enables to directly switch between parabola, rectangular, flat-top or even dark parabola temporal intensity profiles by simply varying the spectral phase parameter $\varphi[20]$.

In order to complement the kurtosis excess at $A^{2}=-12 \mathrm{~dB}$ (see figure $2(\mathrm{~b})$ ) and to characterize more accurately the generated pulse shape, we also evaluated the misfit factor $M$ between the synthesized pulse shape $I(t)$ and a fit by the targeted intensity profile $I_{f i t}$ where $I_{f i t}$ can be a parabolic or triangular waveform :

$M^{2}=\int\left(I-I_{f i t}\right)^{2} d t / \int I^{2} d t$

Numerical results are depicted in figure 2, panel (c). Misfit factors below 0.03 are reached for $\varphi=1.52 \mathrm{rad}$ 


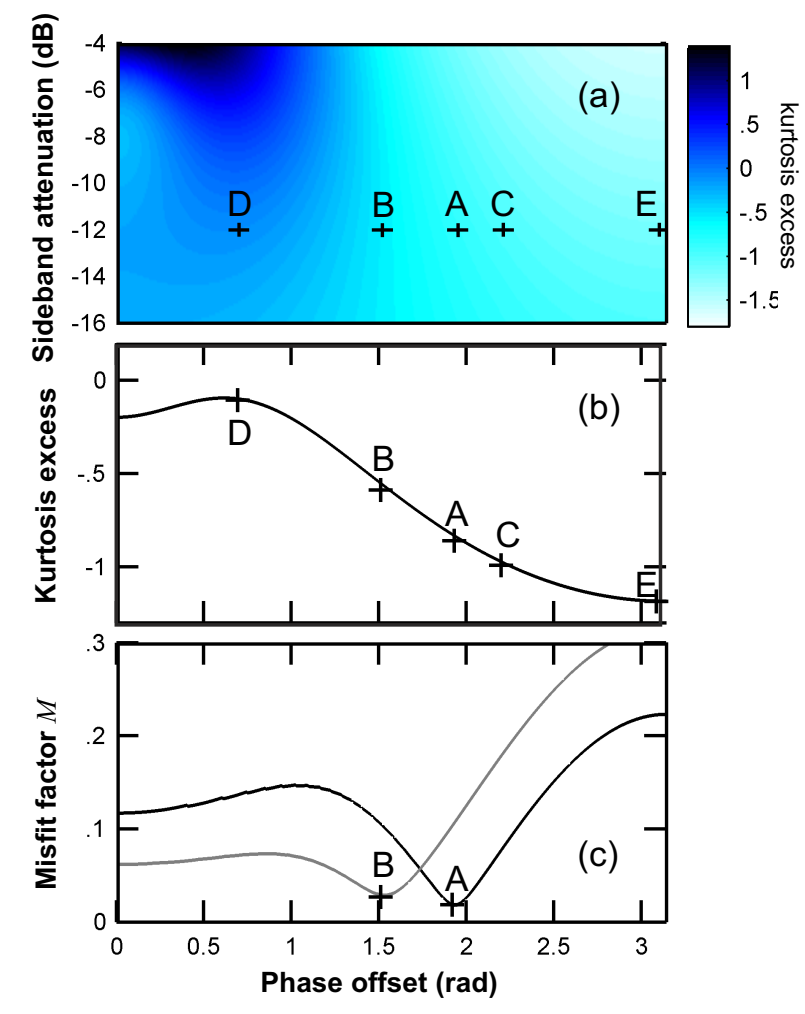

Figure 2. (a) Evolution of the kurtosis excess according to the phase difference $\varphi$ and attenuation $A^{2}$ of the outer sidebands with respect to the inner sidebands. (b) Details of the evolution of the kurtosis excess according to $\varphi$ for a fixed sideband ratio $A^{2}$ of $-12 \mathrm{~dB}$. (c) Evolution of misfit factor $M$ according to $\varphi$ for a fixed sideband ratio $A^{2}$ of $-12 \mathrm{~dB}$ and for a parabolic and triangular target (black and grey lines respectively). Points A, B, C, D and E are the parameters used in subplots (a), (b), (c), (d) and (e) of figure 3 , respectively.

and $\varphi=1.92 \mathrm{rad}$ (triangular and parabolic waveforms respectively, points $B$ and $A$ of figure 2 ) indicate that high quality pulse profiles can be synthesized. Details of the resulting intensity profiles are reported in figure $3(a-b)$ for a repetition rate $R$ of $80 \mathrm{GHz}$ and confirm the very good agreement between the generated profiles and the desired shapes (the fits are plotted with filled grey circles). Nevertheless, the temporal chirp profile, also plotted in figure 3, reveals that the generated pulses are far from being Fourier transform-limited or even linearly chirped. Actually, in contrast to conventional schemes that aim to carve in the spectral domain both temporal and phase profiles, in our bidimensional analysis we do not take into account any constraint on the temporal phase profile for the generated pulses. For other operating points $\mathrm{C}, \mathrm{D}$ and $\mathrm{E}$ (corresponding to $\varphi=2.21$, 0.7 , and 3.1 rad respectively), dark parabolic, flat-top and double-peaked waveforms are respectively generated as illustrated in panels (c-e) of figure 3.
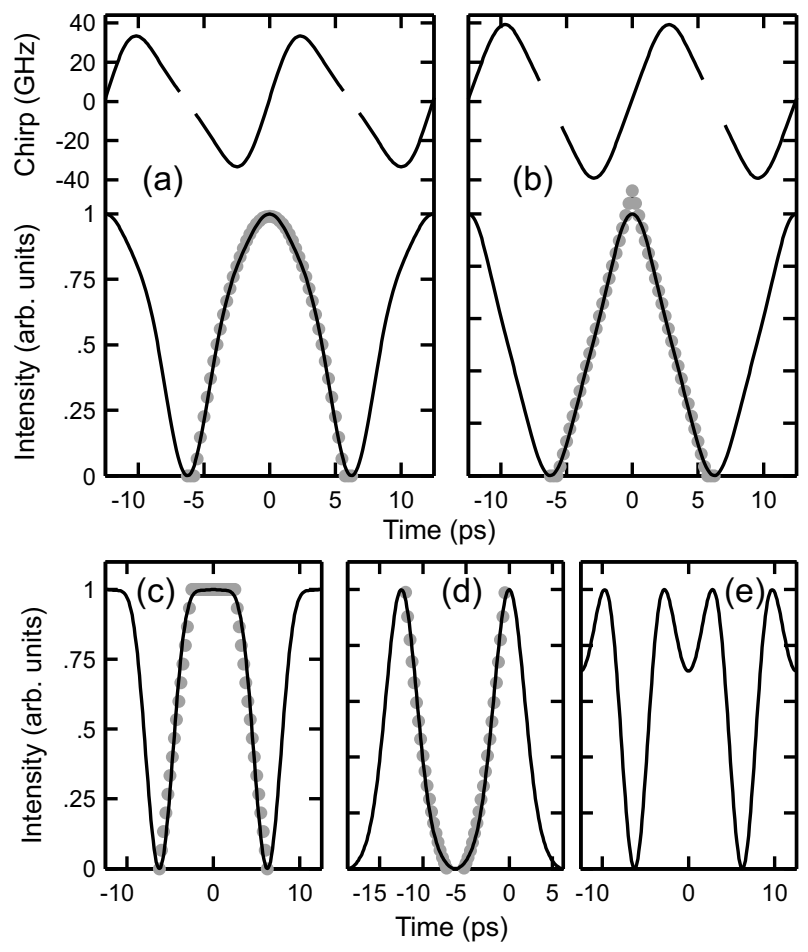

Figure 3. Various pulse shapes of the $80-\mathrm{GHz}$ repetition rate pulse train obtained numerically for a sideband ratio $A^{2}$ of $-12 \mathrm{~dB}$. (a) and (b) Temporal intensity and phase profiles obtained for the points $A$ and $B$ of figure 2 leading to the generation of parabolic and triangular pulse trains respectively. (c),(d) and (e) Other temporal intensity profiles generated for a phase difference $\varphi$ of 2.24, 0.70 and $3.1 \mathrm{rad}$ (points C, D and E) : flat-top pulse, dark parabolic profile, double peaked structure. The intensity profile is compared with a fit by the target waveform (solid grey circles).

\subsection{Generation of the initial four spectral sidebands}

Our approach relies on the phase shaping of a symmetric spectrum made of four spectral lines having a ratio $A^{2}$ of $-12 \mathrm{~dB}$. In a previous proof-of-principle experiment [20], such a spectrum was obtained through the phase modulation of a continuous wave at $20 \mathrm{GHz}$. The approach was not really power efficient as half of the spectral components had to be removed. Moreover, the scaling of this technics to repetition rates higher than $40 \mathrm{GHz}$ becomes technically challenging due to the bandwidth limitation of current optoelectronic devices. To overcome this issue, we propose here an alternative solution that takes advantage of the nonlinear propagation of a sinusoidal beating in an optical fiber.

In order to illustrate such an approach, we first numerically consider the evolution of a carriersuppressed $80-\mathrm{GHz}$ sinusoidal beat-signal in a highly nonlinear fiber having a second order dispersion coefficient of $0.5 \mathrm{ps} / \mathrm{km} / \mathrm{nm}$ and a nonlinear coefficient $\gamma$ of $10 / \mathrm{W} / \mathrm{km}$. We performed numerical simulations of the propagation along the distance $L=1 \mathrm{~km}$ based on the standard nonlinear Schrödinger equation. Four- 
wave mixing process between the two initial spectral components creates new symmetric and regularly spaced sidebands. Figure 4(a) summarizes the evolution of the ratio $A^{2}$ (solid blue line) according to the initial average power $P_{0}$. The targeted ratio of $-12 \mathrm{~dB}$ can be reached for an input power of $100 \mathrm{~mW}$. The results of the numerical simulations are in full agreement with the theoretical prediction of Boskovic et al [22] assuming a pure selfphase modulation effect (blue diamonds) where the ratio $A^{2}$ is predicted by :

$A^{2}=\frac{J_{0}(\phi / 2)^{2}+J_{1}(\phi / 2)^{2}}{J_{1}(\phi / 2)^{2}+J_{2}(\phi / 2)^{2}}$

where $\phi$ is the nonlinear phase given by $\phi=\gamma P_{0} L$. This is consistent with the fact that for this range of parameters, the regime of propagation is mainly driven by the Kerr nonlinearity and contrary to $[8,12,23,24]$ the temporal intensity profile is not here modified upon propagation.

Details of the spectrum generated with $100 \mathrm{~mW}$ pump power are given in figure 4 (b) and reveal that due to multiple four-wave mixing process, additionnal lateral spectral components are generated at $200 \mathrm{GHz}$. However, their level remain below $-30 \mathrm{~dB}$ compared to the level of the inner sidebands and we checked that their presence does not impair the expected reshaping. For this range of parameters, there is no signature of modulation instability that could degrade the coherence of the signal [25]. Another noticeable point is that the spectral components are not in phase by contrast to the spectrum resulting from a pure sinusoidal phase modulation of a continuous wave [26].

\section{Generation of a continuous train of shaped pulses}

We first investigate the generation of a continuous train of shaped pulses at a repetition rate $R$ of $80 \mathrm{GHz}$.

\subsection{Experimental setup}

In order to experimentally validate the feasibility of our approach, we implemented a setup exclusively based on commercially available components operating at telecommunication wavelengths as depicted in figure 5 . A continuous-wave laser (an external-cavity laser diode) is first intensity modulated using a Lithium-Niobate intensity modulator electrically driven by a $40-\mathrm{GHz}$ sinusoidal clock and operating at the point of null-transmission. The modulated signal is then amplified by means of a low noise erbium-doped fiber amplifier (EDFA) followed by a programmable optical bandpass filter that partly removes the amplified spontaneous noise emission and limits the energy contained in the central carrier component.
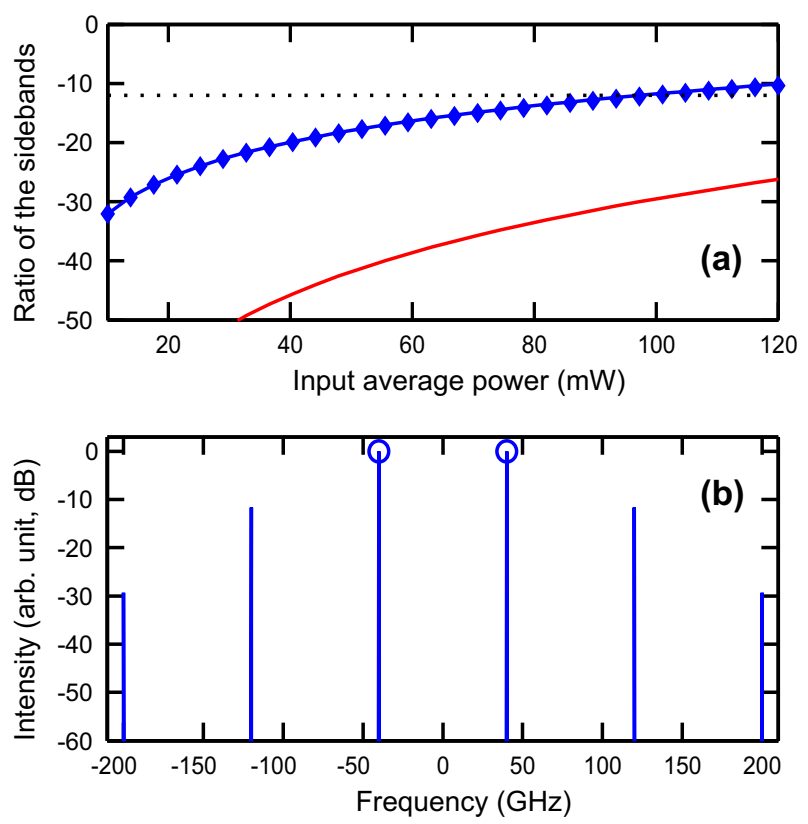

Figure 4. (a) Evolution of the ratio between the intensity of the central and first as well as the second lateral sidebands of the output spectrum according to the input average power (blue and red lines respectively). The dotted line represents the target of $-12 \mathrm{~dB}$. The diamonds represent the analytical predictions [22]. (b) Optical spectrum before and after propagation in a $1-\mathrm{km}$ long HNLF (circle and solid line respectively) for an input average power leading to a $-12 \mathrm{~dB}$ ratio between the central and first lateral sidebands.

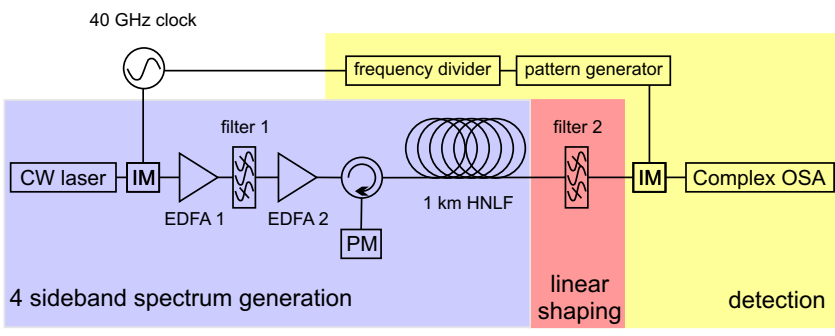

Figure 5. Experimental setup : CW - Continuous Wave, IM - Intensity Modulator, EDFA - Erbium Doped Fiber Amplifier, PM - Power Meter, HNLF - Highly Nonlinear Fiber, OSA - Optical Spectrum Analyser.

A second EDFA is then used to reach the power level required for the generation of the additional pair of lateral sidebands through the self-phase modulation effect occurring in the highly nonlinear fiber whose physical parameters correspond to the one involved in the numerical results described in the above numerical analysis. In order to prevent from the deleterious effects of Brillouin backscattering, an optical circulator is inserted at the fiber input while a powermeter monitors the reflected power. Thereby, the Brillouin threshold has been experimentally evaluated around $60 \mathrm{~mW}$.

After this stage of nonlinear propagation, a second programmable optical filter based on liquid crystal on silicon (LCOS) technology (Finisar waveshaper device) 
[4] is then implemented so as to imprint the targeted shaping on the 4-line comb spectrum. The intensity and chirp characterization is achieved using a complex optical spectrum analyzer (APEX technologies, AP2441) that requires the signal to be periodically modulated at a repetition rate of $2.5 \mathrm{GHz}$ or $625 \mathrm{MHz}$. In order to fulfill this technological requirement, we frequency divided the initial $40-\mathrm{GHz}$ clock to obtain a $10-\mathrm{GHz}$ clock that then drives a pattern generator delivering a 1011 pattern at 10 $\mathrm{GHz}$.

\subsection{Experimental results}

The spectrum recorded at the input of the HNLF with the high resolution optical spectrum analyzer is plotted in figure 6(a). As expected from an intensity modulator operating at its null transmission point, the spectrum of the resulting carrier suppressed signal clearly exhibits two components that are separated by twice the initial clock frequency and that are symmetrically located with respect to a residual central spectral line (the extinction ratio of this unwanted component is higher than $30 \mathrm{~dB}$ ).

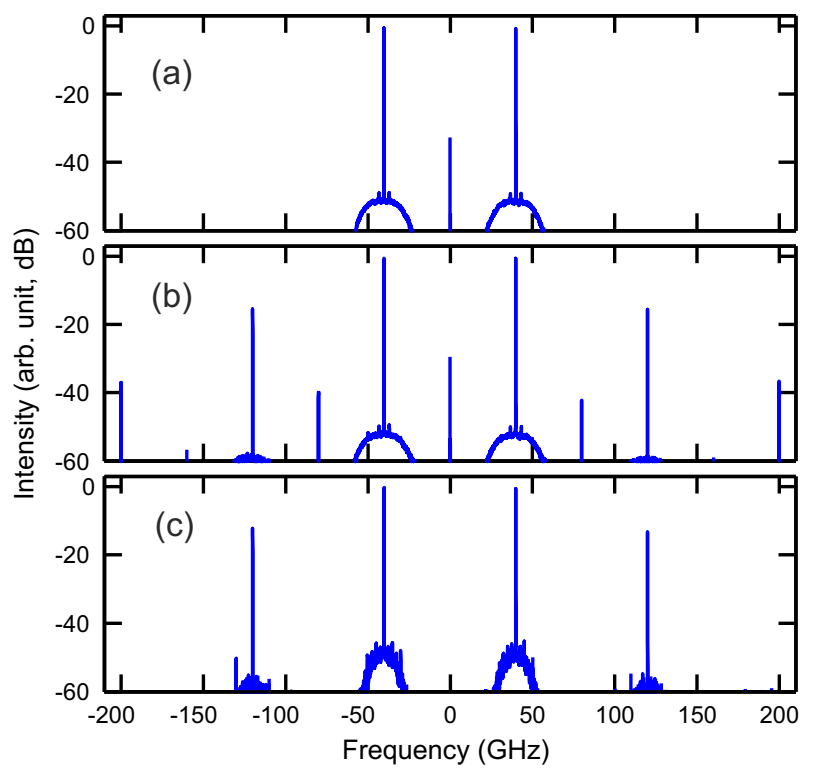

Figure 6. Optical spectrum recorded (a) before the HNLF, (b) after the HNLF and (c) after filter 2. Frequencies of the optical spectra are relative to the carrier frequency $(193.4 \mathrm{THz})$

The expanded spectrum that is obtained after propagation in the HNLF for an input power of $60 \mathrm{~mW}(17.8$ $\mathrm{dBm})$ is plotted in figure $6(\mathrm{~b})$. The expected new lateral sidebands are clearly visible. Given the power limitation associated to the Brillouin scattering, the ratio between the main inner and outer sidebands is limited to $-15.6 \mathrm{~dB}$, which is fully consistent with the numerical simulations discussed in section 2. Note that several approaches such as phase modulation of the input pump [27], longi- tudinal strain or temperature distribution [28-30], as well as aluminum-doped HNLF [31] may be implemented to overcome the Brillouin limitation. We also tested other highly nonlinear fibers with same length and nonlinearity but distinct normal and anomalous dispersion values. Similar results were obtained, thus confirming that selfphase modulation is here the main process driving the spectral expansion.

The spectrum obtained after linear spectral shaping is displayed in figure 6(c). By selectively adding a 3.6- $\mathrm{dB}$ attenuation on the inner sidebands, we achieved the targeted $12-\mathrm{dB}$ ratio. We also removed the residual central line as well as the other spurious sidebands in order to only keep the four significant spectral components we need and to limit pulse to pulse fluctuations. An optical signal-to-noise ratio better than $47 \mathrm{~dB}$ is then achieved. Note that at this stage of linear shaping, the programmable optical filter also imprints the phase difference between the various spectral components.

Results of the temporal characterization obtained for various phase detunings $\varphi$ are presented in figure 7 and demonstrate the generation of the expected pulse shapes at a repetition rate of $80 \mathrm{GHz}$. For illustration, high-quality parabolic profiles are successfully achieved (Figure $7(\mathrm{a})$ ), as well as triangular pulses (Figure $7(\mathrm{~b})$ ). The intensity profiles are in close agreement with the same parabolic or triangular fits as the one used in figure 3).The chirp profiles can also be compared with the ones predicted by numerical simulations and exhibit the same trends. It is noteworthy that simply changing $\varphi$ is here sufficient to switch from one pulse shape to another one , which makes the present method easily reconfigurable and of a high practical interest. The generation of trains of pulses with a high level of flatness can also be observed in figure 7 (c), as well as a train of dark parabola (Figure $7(\mathrm{~d})$ ) or double peaked structure (Figure $7(\mathrm{e})$ ).

\section{Generation of a bunch of shaped pulses}

After the demonstration of a continuous train of shaped pulses at $80 \mathrm{GHz}$, we now investigate the generation of a train of shaped pulses with a temporal finite extend. For this purpose, we modified the experimental setup of figure 5 into the setup sketched in figure 8(a). More precisely, after the generation of the initial sinusoidal beating, the second optical intensity modulator selects a sequence of 8 oscillations. This bunch of pulses is repeated at a rate of $625 \mathrm{MHz}$, leading to a $12 \mathrm{~dB}$ reduction in terms of average power at the input of the HNLF. With such a power reduction, it was possible to reach a ratio of $-12 \mathrm{~dB}$ between the main inner and outer sideband without suffering from any Brillouin backscattering. Con- 

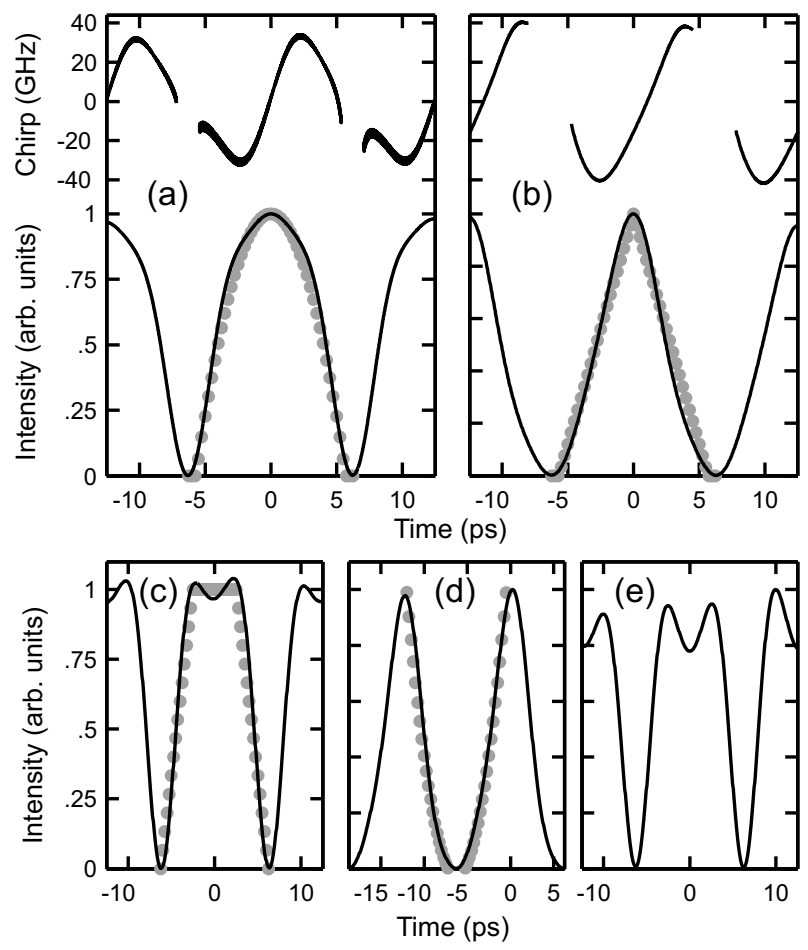

Figure 7. Various pulse shapes obtained experimentally for a spectral ratio $A^{2}$ of $-12 \mathrm{~dB}$ and plotted over two periods. (a) and (b) Temporal intensity and phase profiles corresponding to parabolic and triangular pulse trains respectively. (c),(d) and (e) Other temporal intensity profiles obtained experimentally : flat-top pulse, dark parabolic profile, double peaked structure. The intensity profile is compared with the same fit as the one used in figure 3 (solid grey circles). The small pulseto-pulse fluctuations that are observed may be partly attributed to the detection setup that requires the use of a second intensity modulator.

sequently, the spectral intensity reshaping by the second programmable filter is then no longer required. Only the phase shaping of the 4 -line comb spectrum was here exploited. Note that further optimization of the setup should also enable to get rid of the second amplifier stage.

In this configuration, as shown in figure $8(\mathrm{~b})$ and contrary to the results obtained in the case of a continuous pulse train, the sidebands are no longer composed of a single spectral line. Indeed, the repetition rate of the bunch leads to a comb structure of each sideband with spectral spacing of $625 \mathrm{MHz}$. The spectral extend of the sidebands is directly linked to the duration of the bunch of pulses. Note that with 8 pulses included within a bunch, the spectral width of each sideband is much lower than the repetition rate of the pulse source $(80 \mathrm{GHz})$, leading to well separated sidebands. Since the main four sidebands do not overlap, imprinting a clear phase difference between the inner and outer sidebands can still be experimentally achieved. A set of various temporal intensity profiles that were experimentally synthesized is presented in figure 9 and does not show any degradation compared to the previous case of the shaping of a con- tinuous train.
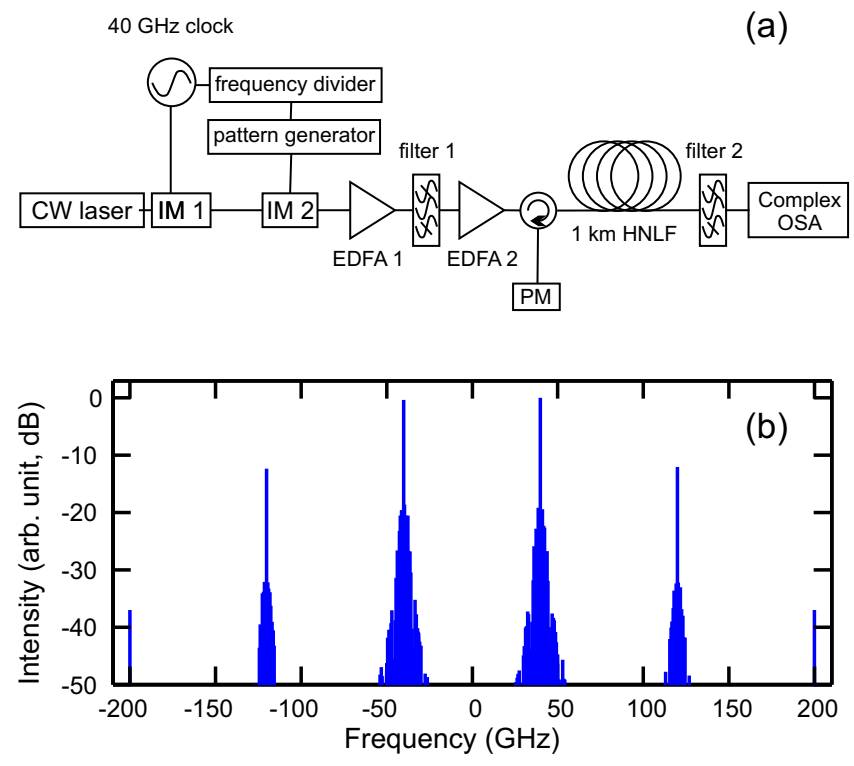

Figure 8. (a)Experimental setup (same acronyms as in figure 5). (b) Experimental spectrum obtained after filter 2 and recorded on a high resolution spectrum.

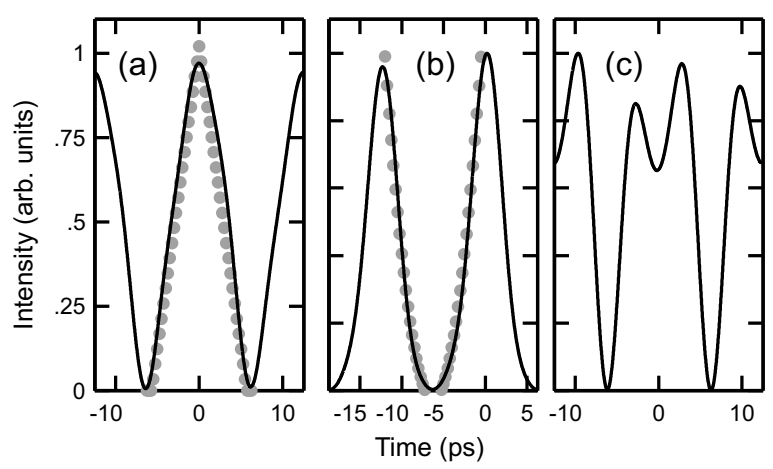

Figure 9. Various pulse shapes obtained experimentally in the case of a bunch of 8 temporal periods : (a) triangular pulse, (b) dark parabolic profile and (c) double peaked structure. The intensity profile is compared with the same fit as the one used in figure 3 and figure 7 (solid grey circles). The small pulse-to-pulse fluctuations that are observed may be partly attributed to the use of two cascaded intensity modulators.

\section{Conclusion}

In conclusion, we have demonstrated the generation of $80-\mathrm{GHz}$ high repetition rate pulse trains based on the linear phase shaping of a single 4-sidebands spectrum. The efficiency as well as the reconfigurable property of this photonic waveform generator was experimentally validated by the various high-quality intensity profiles recorded at $80 \mathrm{GHz}$. In particular, parabolic, triangular 
or flattened waveform intensity shapes as well as their associated chirp profiles were successfully reported and compared with theoretical predictions. Compared to our previous results reported in [20], the present work represents a two-fold improvement in the repetition rate that can be achieved by means of this technic. This progress has been made possible by taking advantage of a four-wave mixing process occurring during the propagation of a $40-\mathrm{GHz}$ beat-signal in a highly nonlinear fiber. More precisely, the self-phase modulation induced by this carrier-suppressed sinusoidal signal generates the outer spectral components with an adequate amplitude ratio with respect to the inner spectral components. We also checked that our method is not only restricted to continuous wave trains and that bunch of shaped pulses could also be synthetized.

In order to provide this first proof-of-principle demonstration, we focused our work on periodic bunches, but it is worth mentioning that periodicity is not required, especially for data-packet applications. Moreover, to obtain the initial bichromatic pump, we here exploit an intensity modulator operating at its null transmission point. However, to reach higher repetition rates and to relax the optoelectronics bandwidth limitation, coherent combination of two continuous signals generated from different lasers [10, 11], bichromatic lasers [32], multi-frequency Brillouin oscillator [33] or microresonator-based generation of stable coherent combs with frequency spacing above 100 $\mathrm{GHz}$ [34] appear as various promising solutions.

\section{Acknowledgments}

We acknowledge the financial support of the Conseil Regional de Bourgogne (Pari Photcom) and the funding of the Labex ACTION program (ANR-11-LABX-0001-01) and the European Regional Development Fund. The experimental work benefited from the PICASSO Platform of the University of Burgundy.

\section{References}

[1] Boscolo S and Finot C 2012 International Journal of Optics 159057

[2] Yao J P 2011 Opt. Commun. 284 3723-36

[3] Cundiff S T and Weiner A M 2010 Nat. Photonics 760-66

[4] Clarke A M, Williams D G, Roelens M A F and Eggleton B J 2010 J. Lightw. Technol. 97-103

[5] Parmigiani F, Petropoulos P, Ibsen M and Richardson D J 2006 IEEE Photon. Technol. Lett. 829-831

[6] Andresen E R, Dudley J M, Finot C, Oron D and Rigneault H 2011 Opt. Lett. 36 707-9

[7] Hirooka T, Nakazawa M and Okamoto K 2008 Opt. Lett. 331102 4

[8] Frisquet B, Chabchoub A, Fatome J, Finot C, Kibler B and Millot G, 2014 Phys. Rev. A 89023821

[9] Chan H S, Hsieh Z M, Liang W H, Kung A, Lee C K, Lai C J, Pan R $P$ and Peng L H, 2011 Science 331 1165-8

[10] D. S. Wu D S, Richardson D J and Slavik R 2015 Optica 2 18-26
[11] Hyodo M, Abedin K and Onodera N 2000 Electron. Lett. 36 224-5

[12] Finot C, Fatome J, Pitois S and Millot G 2007 IEEE Photon. Technol. Lett. 19 1711-3

[13] Boscolo S, Latkin A I and Turitsyn S K 2008 IEEE J. Quantum Electron. 44, 1196-1203

[14] Bale B G, Boscolo S, Hammani K and Finot C 2011 J. Opt. Soc. Am. B 28 2059-65

[15] Jing L, Tigang N, Li P, Wei J, Haidong Y, Hongyao C and Chan Z 2013 IEEE Photon. Technol. Lett. 25 952-4

[16] F. Zhang F, Ge X and Pan S 2013 Opt. Lett. 38 4491-3

[17] Li W, Wang W T, Sun W H, Wang W Y, and Zhu N H 2014 Opt. Express 22 14993-15001

[18] Ye J, Yan L, Pan W, Luo B, Zou X, Yi A and Yao S 2011 Opt. Lett. 36 1458-60

[19] Jiang Y, Ma C, Bai G, Qi X, Tang Y, Jia Z, Zi Y, Huang F and Wu T (2015) Opt. Express 23 19442-52

[20] Finot C 2015 Opt. Lett. 40 1422-5

[21] Kenney F and Keeping E 1951 Mathematics of statistics-part two

[22] Boskovic A, Chernikov S V, Taylor J R, Gruner-Nielsen L and Levring O A 1996 Opt. Lett. 21 1966-8

[23] Fatome J, Finot C, Millot G, Armaroli A and Trillo S 2014, Phys. Rev. X 4021022

[24] Fortier C., Kibler B. , Fatome J. , Finot C., Pitois S. and Millot G. 2008 Laser Phys. Lett. 5 817â€"20

[25] Fatome J, Finot C, Armaroli A and Trillo S 2013 Opt. Lett. 38 181-3

[26] Trillo S, Wabnitz S and Kennedy T 1994 Phys. Rev. A 50 1732-47

[27] Lichtman E, Waarts R G and Friesem A A 1989 IEEE J. Lightwave Technol 7 171-4

[28] Yoshizawa N and Imai T 1993 IEEE J. Lightwave Technol 11 151822

[29] Chavez Boggio J M, Marconi J D and Fragnito H L IEEE J. Lightwave Technol. 23 3808-14.

[30] Imai Y and Shimada N 1993 IEEE Photon. Technol. Lett 5 1335-7

[31] Gruner-Nielsen L, Jakobsen D, Herstrom S, Palsdottir B, Dasgupta S, Richardson D J, Lundstrom C, Olsson S and Andrekson P 2012 38th European Conference and Exhibition on Optical Communications.

[32] Yu L, Zhou D and Zhao L 2014 Laser Phys. Lett. 11095404.

[33] Buttner T F, Kabakova I V, Hudson D D, Pant R, Poulton C G, Judge A C and Eggleton B J 2014 Scientific Reports 45032.

[34] Ferdous F, Miao H, Leaird D E, Srinivasan K, Wang J, Chen L, Varghese L T and Weiner A M 2011 Nat. Photonics 5 770-6 